\title{
Calcificação vascular: fisiopatologia e implicações clínicas
}

\author{
Vascular calcification: pathophysiology and clinical implications
}

\author{
Marcel Liberman ${ }^{1}$, Antonio Eduardo Pereira Pesaro ${ }^{1}$, Luciana Simão Carmo ${ }^{1}$, Carlos Vicente Serrano $\mathrm{Jr}^{2}$
}

\begin{abstract}
RESUMO
A calcificação vascular na doença arterial coronária está ganhando importância, tanto em pesquisas científicas como em aplicações clínicas e de imagem. A placa calcificada é considerada a forma mais relevante de aterosclerose dentro da árvore arterial coronária e frequentemente apresenta um desafio para a intervenção percutânea. Estudos recentes têm demonstrado que a calcificação da placa é dinâmica e está estreitamente ligada ao grau de inflamação vascular. Vários fatores inflamatórios, produzidos durante as diferentes fases da aterosclerose, induzem a expressão e ativação de células osteoblásticas localizadas na parede arterial, que, por sua vez, promovem a deposição de cálcio. As células do músculo liso vascular possuem uma capacidade extraordinária de sofrer diferenciação fenotípica osteoblástica. Não há dúvida de que o papel desses fatores, bem como os elementos de genômica e proteômica, poderia ser um ponto estratégico fundamental na prevenção e no tratamento. Neste contexto, realizamos uma atualização sobre a calcificação coronária, com foco em fisiopatologia, modelos experimentais e implicações clínicas da calcificação vascular.
\end{abstract}

Descritores: Calcificação vascular; Aterosclerose; Isquemia miocárdica; Vitamina D; Insuficiência renal

\section{ABSTRACT}

Vascular calcification in coronary artery disease is gaining importance, both in scientific research and in clinical and imaging applications. The calcified plaque is considered the most relevant form of atherosclerosis within the coronary artery tree and is frequently a challenge for percutaneous intervention. Recent studies showed that plaque calcification is dynamic and is strictly related to the degree of vascular inflammation. Several inflammatory factors produced during the different phases of atherosclerosis induce the expression and activation of osteoblastic cells located within the arterial wall, which, in turn, promote the deposit of calcium. The vascular smooth muscle cells have an extraordinary capacity to undergo osteoblastic phenotypical differentiation. There is no doubt that the role of these factors, as well as the elements of genomics and proteomics, could be a vital strategic point in prevention and treatment. Within this context, we conducted an updating review on coronary calcification focused on pathophysiology, experimental models, and clinical implications of vascular calcification.

Keywords: Vascular calcification; Atherosclerosis; Myocardial ischemia; Vitamin D; Renal insufficiency

\section{INTRODUÇÃO}

A calcificação vascular é um processo fisiopatológico relevante e que se correlaciona com a aterosclerose coronária, sendo um marcador prognóstico de morbimortalidade cardiovascular ${ }^{(1-3)}$. A calcificação determina alterações fisiopatológicas importantes do envelhecimento vascular ${ }^{(4)}$, como, por exemplo, a diminuição da complacência vascular e a elevação da pressão de pulso (por aumentar a pressão arterial sistólica e diminuir a diastólica), além de alterações distributivas do fluxo e perda de vários mecanismos de autorregulação.

O objetivo desta revisão foi explorar os aspectos fisiopatológicos da calcificação vascular, por meio da apresentação de condições clínicas, laboratoriais e epidemiológicas mais prevalentes e, assim, discutir os principais mecanismos celulares e os mediadores que participam deste processo.

\section{IMPORTÂNCIA EPIDEMIOLÓGICA E ASSOCIAÇÃO COM MORTALIDADE CARDIOVASCULAR}

A calcificação vascular, previamente considerada uma condição degenerativa passiva do envelhecimento (Monckeberg 1904), é caracterizada, na atualidade, como um processo de biomineralização ativo ${ }^{(5)}$ e complexamente regulado $^{(6)}$, semelhante à osteogênese $\mathrm{s}^{(7)}$. No

\footnotetext{
Hospital Israelita Albert Einstein, São Paulo, SP, Brasil.

2 Instituto do Coração, Hospital das Clínicas, Faculdade de Medicina, Universidade de São Paulo, São Paulo, SP, Brasil.

Autor correspondente: Marcel Liberman - Avenida Albert Einstein, 627/701, bloco A, 2osubsolo - Morumbi - CEP: 05652-900 - São Paulo, SP, Brasil - Tel.: (11) 2151-1338 - E-mail: malib@einstein.br

Data de submissão: 22/4/2013 - Data de aceite: 22/8/2013
} 
cenário epidemiológico, a calcificação vascular aumenta com a idade, aterosclerose, insuficiência renal ${ }^{(8)}$, diabetes mellitus, hipercolesterolemia, osteoporose, obesidade, tabagismo, menopausa e falta de atividade física ${ }^{(9)}$. A calcificação da aorta pode chegar a $65 \%$ em uma população geral com idade média de 60 anos e se correlaciona à calcificação coronariana detectada por tomografia computadorizada de multidetectores, possuindo valor preditivo positivo quanto à morbidade e à mortalidade cardiovascular em pacientes assintomáticos com risco intermediário ${ }^{(2)}$. Ainda, a calcificação da aorta abdominal está associada à mortalidade cardiovascular aumentada, mesmo quando ajustada para idade ${ }^{(2)}$. Por outro lado, a calcificação de artérias coronárias se correlaciona com maior risco de infarto do miocárdio e com incidência aumentada de eventos adversos durante a revascularização miocárdica percutânea e cirúrgica ${ }^{(3)}$.

A esclerose valvar aórtica, que possui $40 \%$ de prevalência em pacientes octogenários ${ }^{(9)}$, é um processo inicial da estenose valvar aórtica calcificada, em que a mineralização da cúspide tem fisiopatologia semelhante à calcificação vascular ${ }^{(10)}$, caracterizando-se com preditor de risco cardiovascular no idoso ${ }^{(11)}$. Ao contrário de valvas controles, aquelas com calcificação expressam mais fosfatase alcalina e metaloproteinase- 2 de matriz $^{(12)}$. Apesar da degeneração calcificada da valva aórtica se associar à aterosclerose e a seus fatores de risco, estudos que investigaram o uso de estatinas em pacientes com estenose valvar aórtica não demonstraram diminuição da progressão da doença ${ }^{(13)}$.

\section{BASES FISIOPATOLÓGICAS E CLÍNICAS DA CALCIFICAÇÃO VASCULAR}

A insuficiência renal crônica (IRC), o diabetes mellitus e a aterosclerose são as condições clínicas que, epidemiologicamente, mais colaboram para o desenvolvimento da calcificação vascular nas camadas média e íntima do vaso (Quadro 1). No mundo real, porém, a calcificação vascular ocorre indistintamente nessas túnicas vasculares, com diferentes espectros devido a variabilidade, intensidade e coexistência de fatores de risco e de doença na população. Na tentativa de tornar a discussão mais didática, expomos aqui a contribuição de algumas comorbidades, fatores de risco e respectivas alterações clínico-laboratoriais que podem implicar na fisiopatologia da calcificação vascular, apesar de, muitas vezes, tais condições coexistirem na população e ainda se constituírem de fatores de risco intercambiáveis entre si, como é o caso do diabetes em relação à aterosclerose, e à IRC e vice-versa. Assim, a etiologia mais frequente da IRC é a glomerulosclerose diabética,
Quadro 1. Formas de calcificação vascular, manifestações clínicas mais frequentes e fatores de risco na população em geral e com insuficiência renal crônica ${ }^{(8)}$

\begin{tabular}{|c|c|c|c|}
\hline Lesão & $\begin{array}{c}\text { Manifestação } \\
\text { clínica }\end{array}$ & $\begin{array}{c}\text { Possíveis fatores de } \\
\text { risco na população } \\
\text { geral }\end{array}$ & $\begin{array}{l}\text { Possíveis fatores } \\
\text { de risco em } \\
\text { pacientes com IRC }\end{array}$ \\
\hline $\begin{array}{l}\text { Aterosclerose: } \\
\text { placas ou } \\
\text { lesões } \\
\text { circunferenciais }\end{array}$ & $\begin{array}{l}\text { Doença arterial } \\
\text { coronária, } \\
\text { arritmia, morte } \\
\text { súbita, doença } \\
\text { vascular } \\
\text { periférica, } \\
\text { estenose da } \\
\text { artéria renal }\end{array}$ & $\begin{array}{l}\text { Predisposição genética, } \\
\text { tabagismo, hipertensão } \\
\text { arterial, diabetes, } \\
\text { dislipidemia, inflamação }\end{array}$ & $\begin{array}{l}\text { Os tradicionais, além } \\
\text { dos distúrbios do } \\
\text { metabolismo ósseo, } \\
\text { produtos de glicação } \\
\text { avançados (AGEs), } \\
\text { estresse oxidativo e } \\
\text { inflamação }\end{array}$ \\
\hline $\begin{array}{l}\text { Calcificação da } \\
\text { média }\end{array}$ & $\begin{array}{l}\text { Aumento da } \\
\text { pressão de } \\
\text { pulso, isquemia } \\
\text { de múltiplos } \\
\text { órgãos, } \\
\text { estenose valvar } \\
\text { aórtica }\end{array}$ & $\begin{array}{c}\text { Diabetes, } \\
\text { envelhecimento }\end{array}$ & $\begin{array}{c}\text { Diabetes, } \\
\text { envelhecimento, } \\
\text { distúrbios do } \\
\text { metabolismo ósseo, } \\
\text { AGEs, estresse } \\
\text { oxidativo e inflamação }\end{array}$ \\
\hline $\begin{array}{l}\text { Arteríolopatia } \\
\text { calcífica } \\
\text { urêmica } \\
\text { (calcifilaxia) }\end{array}$ & $\begin{array}{l}\text { Lesões } \\
\text { isquêmicas de } \\
\text { pele }\end{array}$ & Não observados & $\begin{array}{c}\text { Distúrbios do } \\
\text { metabolismo ósseo, } \\
\text { especialmente } \\
\text { hiperfosfatemia, } \\
\text { obesidade, gênero } \\
\text { feminino e raça branca }\end{array}$ \\
\hline
\end{tabular}

IRC: insuficiência renal crônica.

representando 50\% dos indivíduos com IRC. É uma situação clínica em que prevalecem: o hiperparatireoidismo secundário, a elevação do paratormônio intacto com alteração das concentrações plasmáticas de vitamina D3, cálcio, fósforo e um aumento do produto cálcio e fósforo, graças, principalmente, ao aumento do fósforo ${ }^{(8)}$. Ainda, pacientes com IRC têm diminuição da fetuína A, que inibe a calcificação ectópica. Além disso, pacientes com quartil de produto cálcio e fósforo mais elevado (que per se incrementa a calcificação vascular) tiveram, paralelamente, proteína C-reativa (PCR) mais alta do que aqueles com quartil de produto cálcio e fósforo mais baixo. Isso implica em uma relação direta entre produto cálcio e fósforo, índice inflamatório (PCR) e calcificação vascular $^{(14)}$. Outras patologias em que o produto cálcio e fósforo está elevado, podendo provocar calcificação vascular, são doenças bem mais raras como a doença de Paget (na qual há uma atividade aumentada de osteoclastos, inclusive com fraturas ósseas), o hiperparatireoidismo primário (por tumor primário da glândula paratireoide).

Recentemente, foram demonstradas a presença de proteínas reguladoras e a existência de células desdiferenciadas a partir de células musculares lisas vasculares (VSMCs - vascular smooth muscle cells), chamadas células vasculares calcificadoras $(\mathrm{CVCs})^{(15)}$, implicadas na síntese/reabsorção óssea em placas ateroscleróticas, especialmente ao redor de calcificação ${ }^{(5)}$. Por isso, foi 
proposto que a formação óssea no vaso fosse, em alguns aspectos, semelhante à do osso ${ }^{(7)}$. Contudo, sabemos que, para solucionar o paradoxo de osteoporose/ calcificação vascular, situação clínica muito comum na população geriátrica, na qual existe calcificação vascular em oposição à perda óssea esquelética, certamente a regulação da síntese óssea no vaso e no esqueleto são diferentes. Isso já foi estudado em um modelo in vitro em que osteoblastos do esqueleto e CVCs (população de células da parede do vaso com características osteoblásticas) foram mantidas em cultura e tiveram respostas opostas (diminuição e aumento da secreção óssea, respectivamente) após estímulo com LDL oxidado $\left(\right.$ LDLox $^{(6)}$ ou após estresse oxidativo ${ }^{(16)}$.

Processos inflamatórios crônicos, como é o caso da aterosclerose, podem cursar com calcificação. Isso é desencadeado a partir da resposta à injúria causada pelo LDLox, que inicia o processo inflamatório, amplificado pela exposição de moléculas de adesão, da secreção de interleucinas, PCR e de proteínas morfogênicas de osso (BMPs - bone morphogenetic proteins) pelo endotélio e por células musculares lisas. Notadamente, isso ocorre em topografia vascular com força de cisalhamento oscilatório aumentado, como nas bifurcações vasculares. Por fim, tal fato implica no aumento do estresse oxidativo e na diminuição de inibidores da calcificação, como a proteína glutâmica de matriz (MGP) e a osteopontina (OPN).

Evidências experimentais indicam que a atividade inflamatória pró-aterosclerótica apresenta inter-relação com a modulação osteogênica. Células endoteliais, quando expostas ao LDLox, expressam BMPs. Além disso, o fator de necrose tumoral alfa (TNF- $\alpha$ ) e o interferon gama (IFN- $\gamma$ ) estimulam o endotélio a expressar osteoprotegerina (OPG), o que também é observado a partir de osteoblastos e de células musculares lisas quando estimulados por interleucinas (IL) ${ }^{(17)}$.

Em pacientes com diabetes ou hipertensão, a PCR e a IL-6 se correlacionam com os níveis séricos de $\mathrm{OPG}^{(17)}$. Em recente estudo, os níveis séricos elevados de IL-6 ou reduzidos de IL-8 e IL-13 foram preditores independentes de calcificação arterial coronária $(\mathrm{CAC})^{(18)}$. Outro estudo demonstrou correlação entre a CAC, LDLox e a proteína quimiotática de monócitos-1 $(\mathrm{MCP}-1)^{(19)}$. Por outro lado, uma meta-análise falhou em demonstrar correlação entre alguns marcadores inflamatórios e CAC em pacientes estáveis ${ }^{(18)}$. Além disso, a despeito do potencial efeito anti-inflamatório das estatinas em pacientes com doença arterial coronária $(\mathrm{DAC})^{(20)}$ não está claro se esse benefício pode antagonizar o processo de calcificação vascular ${ }^{(21)}$.

Considerando a potencial inter-relação entre a atividade inflamatória e a regulação das proteínas modu- ladoras de osteogênese (PMOs), podemos supor que pacientes com infarto agudo do miocárdio (IAM) apresentem elevação transitória dos níveis séricos dessas proteínas, como uma resposta de fase aguda. De fato, um estudo recente demonstrou que pacientes com IAM tiveram ascensão dos níveis séricos de OPN, com pico no terceiro dia, atingindo valores mais elevados em comparação com indivíduos saudáveis ${ }^{(22)}$.

De forma recíproca, a PCR elevada em pacientes com IRC demonstra um estado inflamatório acentuado, o que já os estratifica com maior risco cardiovascular ${ }^{(23)}$, denotando outro possível mecanismo de inter-relação fisiopatológica. Ainda, pacientes com história pregressa de infarto do miocárdio e níveis séricos mais elevados de fósforo tiveram maior risco de eventos cardiovascu$\operatorname{lares}^{(24)}$. Finalmente, pacientes da comunidade que tinham fosfatemia em quartil mais alto e que não haviam sofrido infarto do miocárdio, também tiveram risco cardiovascular aumentado quando comparados ao quartil mais baixo de fósforo sérico ${ }^{(25)}$, mesmo ajustando para a idade e outros fatores de risco, inclusive a PCR.

\section{DESDIFERENCIAÇÃO DE CÉLULAS MUSCULARES LISAS VASCULARES EM LINHAGEM OSTEOCONDROGÊNICA}

A calcificação da camada íntima e/ou da média vascular (Quadro 1) também pode ocorrer na ausência de aumento do produto cálcio e fóforo, sendo orquestrada por PMOs, como o aumento de BMP2 e de BMP4, e presença de LDLox, como na aterosclerose, o que implica em diferenciação de osteoblastos (CVC), seja a partir de uma célula muscular lisa, célula mesenquimal ou pericito vascular, com consequente aumento da expressão de RUNX2, Osterix (Figura 1), determinando o incremento da atividade fosfatase alcalina, da produção de osteocalcina e de secreção de matriz óssea ${ }^{(26)}$.

Corroborando a hipótese de um processo ativo e

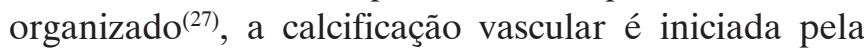
presença de estruturas vesiculares na matriz de tecidos ossificados e de placas ateroscleróticas ${ }^{(26)}$. Essas vesículas de matriz são estruturas ligadas por membranas que se desprendem de condrócitos e osteoblastos, normalmente encontrados em cartilagens e ossos, cuja função é a de nucleação e crescimento de cristais de cálcio. Além disso, a existência de proteínas específicas de osso em placas ateroscleróticas ${ }^{(28)}$, como a OPN, o RUNX2 ou Cbfa-1 (core-binding factor-1, um marcador específico de diferenciação osteoblástica), a OPG, a BMP-2, a MGP, a fosfatase alcalina e o ligante do receptor ativador de NF-kappaB (RANKL), comprova a existência de fatores reguladores in situ de formação e reabsorção óssea no vaso ${ }^{(26)}$. 


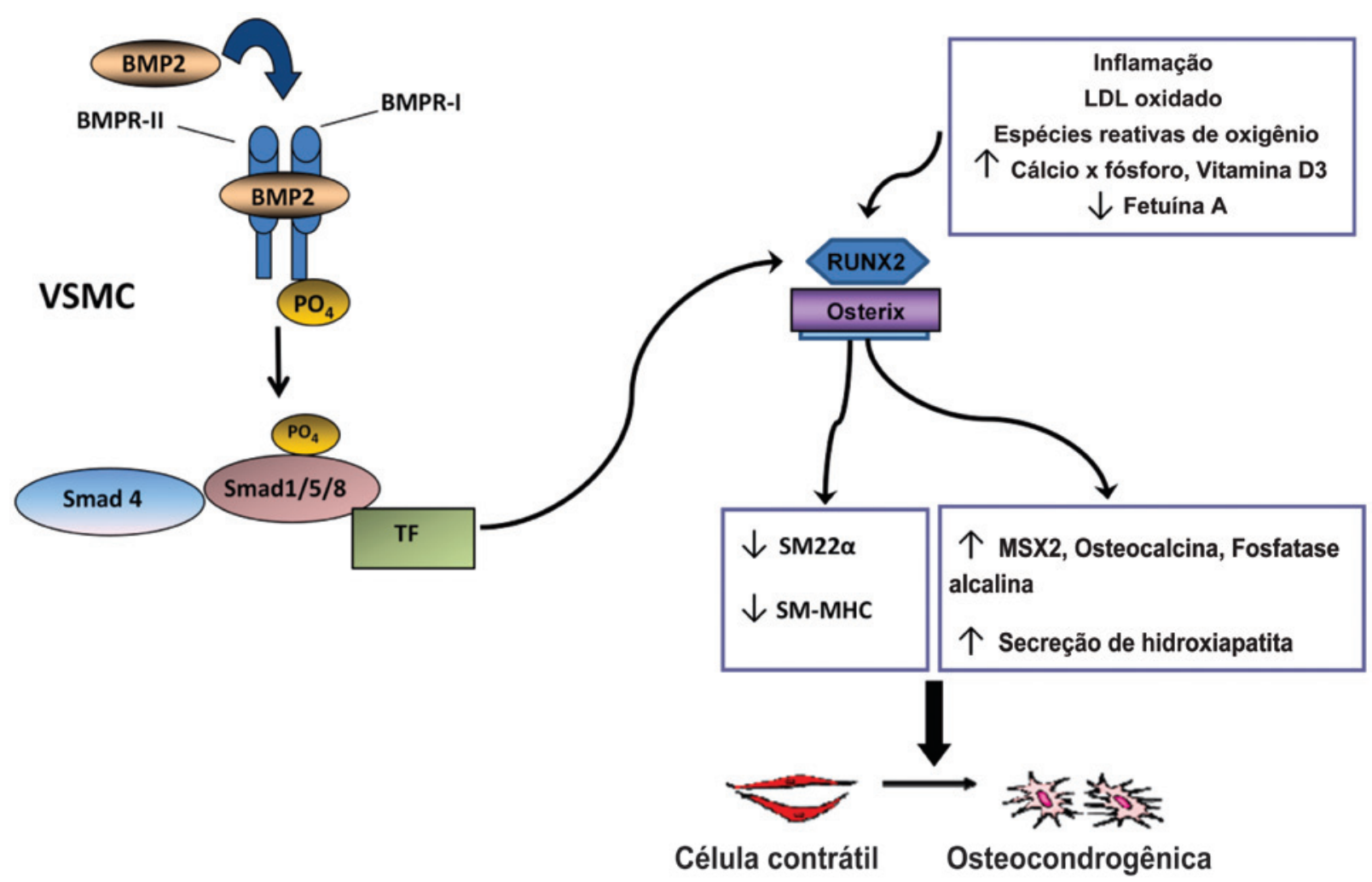

BMP: proteínas morfogênicas de osso; VSMC: células musculares lisas vasculares.

Figura 1. Desdiferenciação de células musculares lisas vasculares em células osteocondrogênicas, resultando em calcificação vascular. BMP2 se liga ao receptor BMPRII, que forma um heterodímero com BMPRI e ativa a sinalização intracelular por meio da fosforilação de Smad1/5/8, formando um complexo com Smad4. No núcleo, há aumento de RUNX2, juntamente de outros fatores de transcrição. Por sua vez, RUNX2 aumenta expressão de Osterix, outro importante modulador estimulatório da calcificação. Em células musculares lisas, esse sinal ativa uma mudança de fenótipo, que pode ser caracterizado por uma diminuição da expressão de marcadores de célula muscular lisa contrátil (SM22a e SM-MHC) e aumento da expressão de marcadores osteocondrogênicos (fosfatase alcalina e osteocalcina), promovendo a secreção de hidroxiapatita. Outros agonistas da calcificação vascular são mostrados à direita como: mediadores inflamatórios, espécies reativas de oxigênio, LDL oxidado, fósforo, vitamina D3 e diminuição da fetuína $\mathrm{A}^{(27,38)}$

As VSMCs incluem subpopulações de células com diferentes fenótipos. Conforme as VSMC em cultura iniciam a diferenciação osteogênica (Figura 1), elas perdem a expressão de marcadores específicos de músculo liso ${ }^{(27)}$, indicando uma desdiferenciação celular. Uma subpopulação de VSMC expressa espontaneamente proteínas de osso $^{(27)}$ e produz uma matriz mineralizada em cultura semelhante à de osteoblastos ${ }^{(7)}$. Essa subpopulação, chamada de $\mathrm{CVC}^{(15)}$ retém a habilidade de se diferenciar em outras linhagens mesenquimais, além de osteoblastos. VSMC humanas também expressam receptor de vitamina D3 e a enzima 25-hidroxivitamina D3-1 $\alpha$-hidroxilase, que pode ter sua expressão aumentada quando são cultivadas com paratormônio (PTH) e compostos estrogênicos ${ }^{(29)}$. Ainda, a desdiferenciação dessas células também depende do cotransportador de fósforo dependente de sódio, Pit-1 ${ }^{(30)}$.

\section{PROTEÍNAS MODULADORAS DA OSTEOGÊNESE}

A diferenciação osteogênica de VSMC é uma etapa central na progressão da calcificação vascular. Essa diferenciação é continuamente modulada por estresse oxidativo, oscilações hormonais e metabólicas e, particularmente, por PMOs. Como descrito melhor adiante, as PMOs podem promover a calcificação vascular diretamente (RANKL e BMPs) ou, eventualmente, serem marcadores da calcificação vascular (OPG e OPN). Adicionalmente, além dos aspectos mecanísticos relacionados às PMOs, há também interesse clínico. Níveis séricos de OPG, por exemplo, já foram identificados como marcadores de $\mathrm{CAC}$ em pacientes com doença coronária estável e como marcadores prognósticos em pacientes com $\operatorname{IAM}^{(31)}$.

Umas das vias de maior interesse e com significado ainda indefinido em relação à fisiopatologia da calcificação vascular é a via $R A N K-R A N K L-O P G$. Ou seja, a interação entre o ativador do receptor do fator nuclear $\kappa B$ (RANK) com seu ligante (RANKL) e ainda a ação inibitória da OPG sobre essa interação. A OPG reduz a atividade do fator nuclear $\kappa B$, fator transcritor de genes imunomediados, e é uma importante reguladora da atividade inflamatória, imunidade inata e diferenciação celular ${ }^{(32)}$. O RANKL é expresso em osteoblastos, 
células musculares lisas, linfócitos T e células estromais, enquanto a OPG é expressa nessas células, além do endotélio.

Em relação ao remodelamento (reabsorção) ósseo, a ativação do RANK pelo RANKL transforma pré-osteoclastos em suas formas maduras ${ }^{(33)}$. Essa interação é diretamente inibida pela OPG. Entretanto, o papel dos osteoclastos na calcificação vascular e na reabsorção óssea no vaso ainda é indeterminado.

Por outro lado, sabidamente o eixo RANK-RANKL$O P G$ participa de diversas etapas de uma complexa cascata inflamatória pró-aterosclerótica (Figura 2). O RANKL é expresso em células musculares lisas e em linfócitos T, modulando a maturação e inibindo a apoptose de células dendríticas. Essa atividade pode ser inibida pela ação da OPG sobre a RANKL. A OPG, por sua vez, é estimulada por uma série de mediadores inflamatórios, tais como IL- 1 , TNF- $\alpha$, TGF- $\beta$ e IFN- $\gamma$. Tais mediadores aumentam a síntese de OPG pelo endotélio. Consequentemente, OPG estimula a expressão de moléculas de adesão e infiltração leucocitária intimal. Esse processo promove a expressão de RANKL, e a proliferação de células musculares lisas ${ }^{(31)}$. RANKL, subsequentemente, estimula diretamente a diferenciação osteogênica dessas células ou, indiretamente, promove a osteogênese via estimulação da secreção de TNF- $\alpha$ em monócitos $^{(34)}$ ou via $\mathrm{BMPs}^{(35)}$. A presença da RANKL em combinação com a OPG (particularmente com uma razão OPG/RANKL reduzida) incrementa a atividade de metaloproteinases, enzimas que participam da erosão e da rotura da capa do ateroma ${ }^{(36)}$.

BMP2, que faz parte da superfamília de TGF- $\beta$, foi originalmente identificado como regulador da formação de cartilagem e do osso. É uma proteína que estimula a diferenciação de osteoblastos ${ }^{(35)}$, e sua presença na placa aterosclerótica sugere que a calcificação arterial é um processo ativamente regulado, semelhante à formação óssea ${ }^{(7,37)}$, como explicado na (Figura 1$)^{(38)}$.

Há pelo menos 20 peptídeos funcionais e estruturais de BMPs relacionados, a maioria com função embriogênica e morfogênica de vários tecidos e órgãos. BMPs ativos biologicamente são usualmente homodímeros contendo uma ligação de cisteína característica em sua estrutura. Os heterodímeros de BMP2/BMP7 e BMP4/BMP7 possivelmente também podem existir in vivo. Os heterodímeros, que podem ter sua atividade mediada por um subtipo de receptor diferente ou adicional, são mais potentes no estímulo de síntese óssea e na indução da mesoderme ventral em relação aos homodímeros. BMPs são proteínas extremamente importantes para o desenvolvimento e diferenciação celular no embrião e filogeneticamente conservadas. BMP é ubiqui-

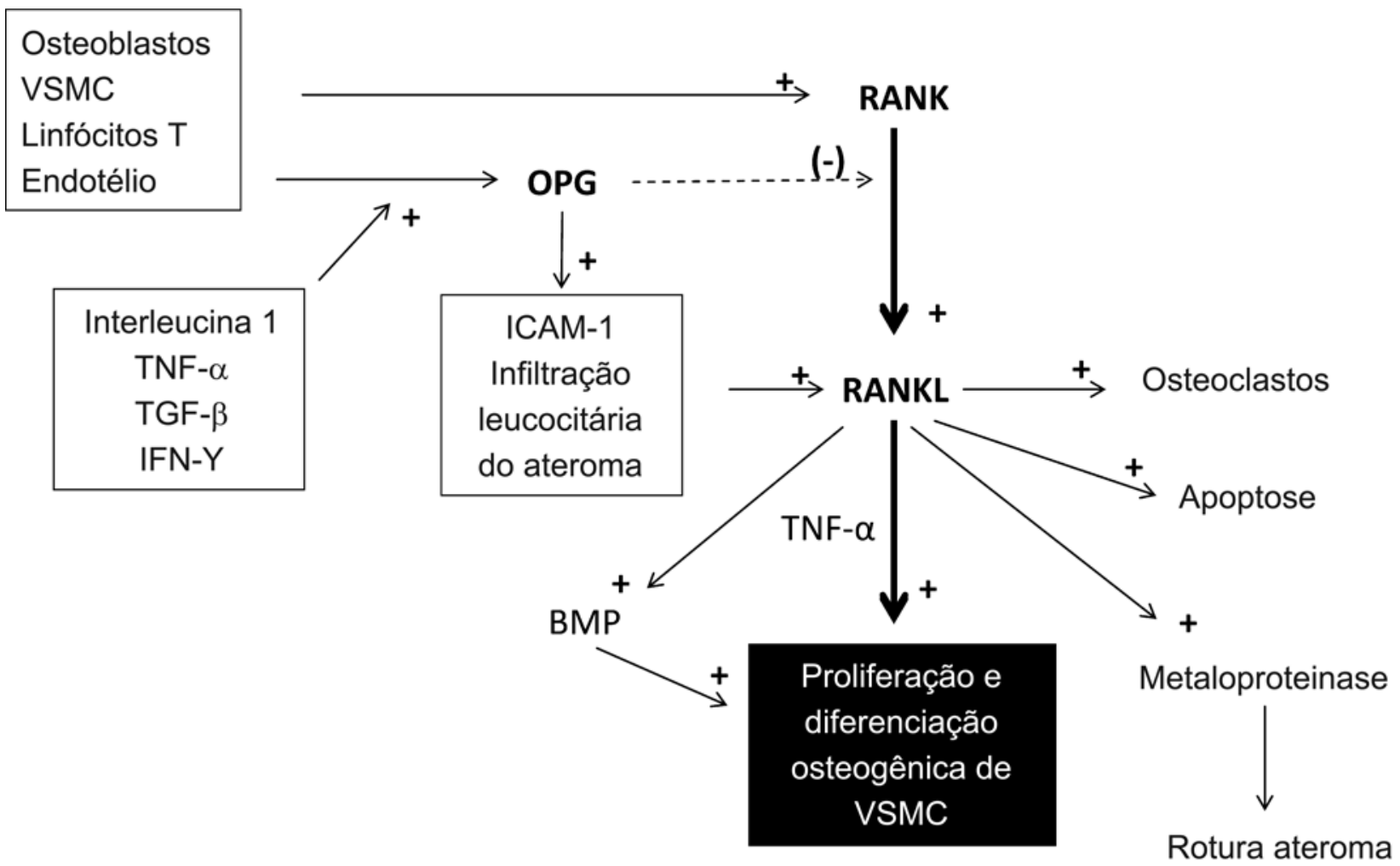

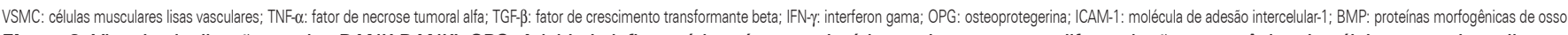
Figura 2. Vias de sinalização no eixo RANK-RANKL-OPG. Atividade inflamatória pró-aterosclerótica pode promover a diferenciação osteogênica de células musculares lisas 
tária em todo reino animal (de insetos a seres humanos) e sua mutação/deleção ou alterações de seu receptor são letais para o desenvolvimento do embrião ${ }^{(26)}$. O aumento da expressão das proteínas ossificadoras sabidamente acontece em células mesenquimais ou mesmo em células pré-miogênicas $\mathrm{C} 2 \mathrm{C} 12$ que tenham seu programa genético de osteoblastogênese ativado por BMP2, por exemplo $^{(39)}$, para se diferenciarem em osteoblastos, em oposição a células musculares lisas, por exemplo, ou a adipócitos. Na parede vascular, os BMPs possivelmente são secretados por múltiplas linhagens celulares: células endoteliais, células musculares lisas, células mesenquimais, pericitos e células inflamatórias ${ }^{(38)}$.

\section{MODELOS EXPERIMENTAIS DE CALCIFICAÇÃO VASCULAR}

O modelo mais difundido de calcificação vascular in vivo, estudado há mais de 40 anos, é baseado na hipervitaminose $\mathrm{D}$ isolada ${ }^{(8)}$. Diversas doses e formas de administração foram descritas, embora a maioria tenha usado curtos períodos de tempo com quantidades potencialmente tóxicas de vitamina $\mathrm{D}$, resultando em hipercalcemia e hiperfosfatemia aguda. O tratamento crônico menos tóxico também resultou em calcificação metastática e deterioração da função renal ${ }^{(8)}$. Em termos gerais, a administração de vitamina D resulta em calcificação da parede arterial e em uma variedade de outras alterações "arterioscleróticas", como a perda de colágeno e o rompimento das fibras de elásticas ${ }^{(4)}$. Essas mudanças são normalmente associadas com aumento da rigidez aórtica ${ }^{(2)}$.

Outro modelo de calcificação vascular envolve a aplicação tópica de $\mathrm{CaCl} 20,5 \mathrm{M}$ na aorta de $\operatorname{coelhos}^{(8,33)}$ ou de outros animais (ratos e camundongos). Os segmentos de artéria submetidos ao $\mathrm{CaCl} 2$ se tornam aneurismáticos, com calcificação da média, inflamação, calcificação da elastina, e degradação e desorganização da matriz extracelular, além de ativação de metaloproteinases de matriz ${ }^{(7,8,33)}$.

$\mathrm{O}$ modelo in vitro de calcificação de VSMCs foi descrito à partir do isolamento de CVCs por meio da cultura de células musculares lisas bovinas, em que nódulos multicelulares apareciam espontaneamente ${ }^{(33)}$. Além de expressarem OPN, osteonectina e colágeno-1, de demonstrarem atividade de fosfatase alcalina aumentada e de produzirem osteocalcina e hidroxiapatita, essas células foram estimuladas a formar um número maior de nódulos de calcificação especialmente quando expostas ao oxisterol 25-hidroxicolesterol, assim como ao TGF $\beta-1$, ambos presentes em placas ateroscleróticas. Esse modelo foi aperfeiçoado por meio da incubação dessas células com um doador de fosfato inorgânico, o $\beta$-glicerofosfato $10 \mathrm{mM}$, resultando em calcificação difusa após 14 dias de cultura ${ }^{(15)}$. A mineralização diminuiu com o uso de levamisol, um inibidor inespecífico da fosfatase alcalina. Além disso, quando $1,25(\mathrm{OH})$-vitamina $\mathrm{D} 3$ foi associado ao $\beta$-glicerofosfato em VSMCs em cultura, a calcificação aumentou progressivamente com incremento da dose, principalmente pela diminuição da expressão do peptídeo endógeno semelhante ao hormônio da paratiroide (PTHrP) nessas células, que é um inibidor endógeno da calcificação vascular $^{(33)}$. Além disso, o efeito estimulatório da 1,25(OH)vitamina D3, na atividade da fosfatase alcalina e a expressão da OPN, podem contribuir para sua ação promotora da calcificação vascular. Pit-1 é um cotransportador de fosfato dependente de sódio do tipo III, muito importante para a mineralização de células incubadas em meio rico em fosfato ${ }^{(30)}$. Expandimos tal conhecimento demonstrando que o BMP2, que está presente na placa de aterosclerose ${ }^{(35,38,40)}$, estimula a calcificação de células musculares lisas de coronárias humanas em cultura, por meio de sinalização específica (Figura 1), perda de marcadores de célula muscular lisa e aumento de expressão de proteínas osteocondrogênicas ${ }^{(37-40)}$.

\section{CONSIDERAÇÕES FINAIS}

A diminuição da progressão da calcificação vascular depende da abordagem terapêutica de múltiplas vias fisiopatológicas. Certamente, é importante diminuir o impacto e controlar os fatores de risco cardiovasculares ${ }^{(9)}$ como dislipidemia, diabetes mellitus, hipertensão arterial sistêmica, insuficiência renal e uremia, além do desbalanço do metabolismo do cálcio e fósforo. Alguns estudos demonstram que estatinas, vitamina $\mathrm{D}$ e seus análogos, e bisfosfonatos parecem diminuir a progressão da doença em determinados grupos de pacientes ${ }^{(8,13,21,26,33)}$.

\section{CONCLUSÕES}

Atualmente, a calcificação vascular é compreendida por meio de mecanismo fisiopatológico complexamente regulado, principalmente desencadeado quando há um desequilíbrio, predominando proteínas e fatores de transcrição de síntese em prejuízo aos mediadores de reabsorção óssea. O melhor entendimento dessa fisiopatologia é essencial para que novas propostas terapêuticas e potenciais marcadores prognósticos sejam explorados, a fim de diminuir a progressão da calcificação vascular.

\section{REFERÊNCIAS}

1. Raggi P, Callister TQ, Cooil B, He ZX, Lippolis NJ, Russo DJ, et al. Identification of patients at increased risk of first unheralded acute myocardial infarction by electron-beam computed tomography. Circulation. 2000;101(8):850-5. 
2. Wilson PW, Kauppila LI, O'Donnell CJ, Kiel DP, Hannan M, Polak JM, et al. Abdominal aortic calcific deposits are an important predictor of vascular morbidity and mortality. Circulation. 2001;103(11):1529-34.

3. Wayhs R, Zelinger A, Raggi P. High coronary artery calcium scores pose an extremely elevated risk for hard events. J Am Coll Cardiol. 2002;39(2):225-30.

4. Roberts WC. The senile cardiac calcification syndrome. Am J Cardiol. 1986; 58(6):572-4

5. Shanahan CM, Cary NR, Metcalfe JC, Weissberg PL. High expression of genes for calcification-regulating proteins in human atherosclerotic plaques. J Clin Invest. 1994;93(6):2393-402.

6. Demer LL. Vascular calcification and osteoporosis: inflammatory responses to oxidized lipids. Int J Epidemiol. 2002;31(4):737-41

7. Boström K, Watson KE, Stanford WP, Demer LL. Atherosclerotic calcification: relation to developmental osteogenesis. Am J Cardiol. 1995;75(6):88B-91B.

8. Wolisi GO, Moe SM. The role of vitamin D in vascular calcification in chronic kidney disease. Semin Dial. 2005;18(4):307-14.

9. Aronow WS, Schwartz KS, Koenigsberg M. Correlation of serum lipids, calcium, and phosphorus, diabetes mellitus and history of systemic hypertension with presence or absence of calcified or thickened aortic cusps or root in elderly patients. Am J Cardiol. 1987;59(9):998-9.

10. Liberman M, Bassi E, Martinatti MK, Lario FC, Wosniak J, Jr., Pomerantzeff $\mathrm{PM}$, et al. Oxidant generation predominates around calcifying foci and enhances progression of aortic valve calcification. Arterioscler Thromb Vasc Biol. 2008;28(3):463-70.

11. Otto CM, Lind BK, Kitzman DW, Gersh BJ, Siscovick DS. Association of aorticvalve sclerosis with cardiovascular mortality and morbidity in the elderly. N Engl J Med. 1999;341(3):142-7.

12. Jian B, Jones PL, Li Q, Mohler ER 3rd, Schoen FJ, Levy RJ. Matrix metalloproteinase- 2 is associated with tenascin- $C$ in calcific aortic stenosis. Am J Pathol. 2001;159(1):321-7.

13. Cowell SJ, Newby DE, Prescott RJ, Bloomfield P, Reid J, Northridge DB, Boon NA; Scottish Aortic Stenosis and Lipid Lowering Trial, Impact on Regression (SALTIRE) Investigators. A randomized trial of intensive lipid-lowering therapy in calcific aortic stenosis. N Engl J Med. 2005;352(23):2389-97.

14. Movilli E, Feliciani A, Camerini C, Brunori G, Zubani R, Scolari F, et al. A high calcium-phosphate product is associated with high C-reactive protein concentrations in hemodialysis patients. Nephron Clin Pract. 2005;101(4): c161-7.

15. Shioi A, Nishizawa $Y$, Jono S, Koyama H, Hosoi M, Morii H. Betaglycerophosphate accelerates calcification in cultured bovine vascular smooth muscle cells. Arterioscler Thromb Vasc Biol. 1995;15(11):2003-9.

16. Mody N, Parhami F, Sarafian TA, Demer LL. Oxidative stress modulates osteoblastic differentiation of vascular and bone cells. Free Radic Biol Med. 2001;31(4):509-19.

17. Cola C, Almeida M, Li D, Romeo F, Mehta JL. Regulatory role of endothelium in the expression of genes affecting arterial calcification. Biochem Biophys Res Commun. 2004;320(2):424-7.

18. Hamirani YS, Pandey S, Rivera JJ, Ndumele C, Budoff MJ, Blumenthal RS, et al. Markers of inflammation and coronary artery calcification: a systematic review. Atherosclerosis. 2008;201(1):1-7.

19. Godsland IF, Pavitt D, Okoturo O, Edwards RJ, Rubens MB, Feher MD, Flather MD, Elkeles RS; PREDICT Study Group. Can protein biomarkers provide an index of coronary artery calcification in patients with Type 2 diabetes? Atherosclerosis. 2010;213(2):570-2

20. Pesaro AE, Serrano CV Jr, Fernandes JL, Cavalcanti AB, Campos AH, Martins HS, et al. Pleiotropic effects of ezetimibe/simvastatin vs. high dose simvastatin. Int J Cardiol. 2012;158(3):400-4.

21. Henein MY, Owen A. Statins moderate coronary stenoses but not coronary calcification: results from meta-analyses. Int J Cardiol. 2011;153(1):31-5.
22. Okyay K, Tavil Y, Sahinarslan A, Tacoy G, Turfan M, Sen N, et al. Plasma osteopontin levels in prediction of prognosis in acute myocardial infarction. Acta Cardiol. 2011;66(2):197-202.

23. Ridker PM, Glynn RJ, Hennekens $\mathrm{CH}$. C-reactive protein adds to the predictive value of total and $\mathrm{HDL}$ cholesterol in determining risk of first myocardial infarction. Circulation. 1998;97(20):2007-11.

24. Tonelli M, Sacks F, Pfeffer M, Gao Z, Curhan G; Cholesterol And Recurrent Events Trial Investigators. Relation between serum phosphate level and cardiovascular event rate in people with coronary disease. Circulation. 2005 112(17):2627-33.

25. Dhingra R, Sullivan LM, Fox CS, Wang TJ, D'Agostino RB Sr, Gaziano JM, et al. Relations of serum phosphorus and calcium levels to the incidence of cardiovascular disease in the community. Arch Intern Med. 2007;167(9):879-85.

26. Doherty TM, Fitzpatrick LA, Inoue D, Qiao JH, Fishbein MC, Detrano RC, et al. Molecular, endocrine, and genetic mechanisms of arterial calcification. Endocr Rev. 2004;25(4):629-72.

27. Steitz SA, Speer MY, Curinga G, Yang HY, Haynes P, Aebersold R, et al. Smooth muscle cell phenotypic transition associated with calcification: upregulation of Cbfa1 and downregulation of smooth muscle lineage markers. Circ Res. 2001;89(12):1147-54.

28. Dhore CR, Cleutjens JP, Lutgens E, Cleutjens KB, Geusens PP, Kitslaar PJ, et al. Differential expression of bone matrix regulatory proteins in human atherosclerotic plaques. Arterioscler Thromb Vasc Biol. 2001;21(12):1998-2003.

29. Somjen D, Weisman $Y$, Kohen F, Gayer B, Limor R, Sharon 0 , et al 25-hydroxyvitamin D3-1alpha-hydroxylase is expressed in human vascular smooth muscle cells and is upregulated by parathyroid hormone and estrogenic compounds. Circulation. 2005;111(13):1666-71.

30. Li X, Yang HY, Giachelli CM. Role of the sodium-dependent phosphate cotransporter, Pit-1, in vascular smooth muscle cell calcification. Circ Res. 2006;98(7):905-12.

31. Omland T, Ueland T, Jansson AM, Persson A, Karlsson T, Smith C, et al Circulating osteoprotegerin levels and long-term prognosis in patients with acute coronary syndromes. J Am Coll Cardiol. 2008;51(6):627-33.

32. Krakauer T. Nuclear factor-kappaB: fine-tuning a central integrator of diverse biologic stimuli. Int Rev Immunol. 2008;27(5):286-92.

33. Abedin M, Tintut Y, Demer LL. Vascular calcification: mechanisms and clinical ramifications. Arterioscler Thromb Vasc Biol. 2004;24(7):1161-70.

34. Tintut $Y$, Patel J, Parhami F, Demer LL. Tumor necrosis factor-alpha promotes in vitro calcification of vascular cells via the cAMP pathway. Circulation. 2000;102(21):2636-42.

35. Zebboudj AF, Shin V, Boström K. Matrix GLA protein and BMP-2 regulate osteoinduction in calcifying vascular cells. J Cell Biochem. 2003;90(4):756-65.

36. Sandberg WJ, Yndestad A, Øie E, Smith C, Ueland T, Ovchinnikova O, et al. Enhanced T-cell expression of RANK ligand in acute coronary syndrome: possible role in plaque destabilization. Arterioscler Thromb Vasc Biol. 2006: 26(4):857-63.

37. Liberman M, Johnson RC, Handy DE, Loscalzo J, Leopold JA. Bone morphogenetic protein-2 activates NADPH oxidase to increase endoplasmic reticulum stress and human coronary artery smooth muscle cell calcification. Biochem Biophys Res Commun. 2011;413(3):436-41.

38. Ryoo HM, Lee MH, Kim YJ. Critical molecular switches involved in BMP2-induced osteogenic differentiation of mesenchymal cells. Gene. 2006; 366(1):51-7.

39. Li Z, Hassan MQ, Volinia S, van Wijnen AJ, Stein JL, Croce CM, et al. A microRNA signature for a BMP2-induced osteoblast lineage commitment program. Proc Natl Acad Sci U S A. 2008;105(37):13906-11.

40. Willette RN, Gu JL, Lysko PG, Anderson KM, Minehart H, Yue T. BMP-2 gene expression and effects on human vascular smooth muscle cells. J Vasc Res. 1999;36(2):120-5. 